\title{
Stress first myocardial perfusion imaging: Is it time to put to rest the "Rest First" strategy for most patients?
}

\author{
Karthik Ananthasubramaniam, MD, FACC, FASE, FASNC, FRCP(Glas) and \\ Sabha Bhatti, MD, FACC
}

\section{See related article, pp. 1124-1134}

While myocardial perfusion imaging (MPI) remains the most validated and extensively utilized modality in the evaluation and prognostication of coronary artery disease (CAD), it has come under unprecedented scrutiny in the past few years both due to the expansive growth of cardiac imaging ${ }^{1}$ and increasing concerns of patient radiation exposure over their lifetime. In one study, nuclear cardiac imaging was reported to be contributing up to $22 \%$ of radiation exposure amongst all imaging. $^{2}$

Over the past few years, major cardiac societies such as the American College of Cardiology/American Heart Association in collaboration with many other imaging societies have taken proactive steps to guide the use of cardiac imaging. Appropriate use criteria for nuclear cardiology, echocardiography, CT/MRI, and most recently, cardiac catheterization and percutaneous coronary interventions have been published to ensure that clinicians in most instances are "doing the right test for the right patient at the right time." Specifically ASNC has been a leader in this effort and has taken numerous initiatives including publication of important documents with practical importance in JNC toward educating physicians regarding the appropriate use of MPI. The recent comprehensive ASNC preferred practice

From the Heart and Vascular Institute, Henry Ford Hospital, Detroit, MI.

Research grant support/speakers bureau: Astellas Pharma US Inc (KA), Lantheus Medical Imaging.

Reprint requests: Karthik Ananthasubramaniam, MD, FACC, FASE, FASNC, FRCP(Glas), Heart and Vascular Institute, Henry Ford Hospital, K-14, Detroit, MI 48202; kananth1@hfhs.org.

J Nucl Cardiol 2012;19:1106-9.

$1071-3581 / \$ 34.00$

Copyright $(\subset) 2012$ American Society of Nuclear Cardiology.

doi:10.1007/s12350-012-9623-9 statement, "Patient Centered Imaging" by Depuey et al ${ }^{3}$ outlines various MPI protocols with existing isotopes and their advantages disadvantages and best use in daily clinical practice. This document reiterates an important point which physicians practicing nuclear cardiology should embrace namely "One size does not fit all."

Most nuclear laboratories perform single day lowdose rest-first, followed by high-dose stress imaging. This enables higher count statistics in the stress portion enabling better images and reliable gating but comes with the disadvantage of lasting about 4 hours and delivering a cumulative radiation dose of $12-15 \mathrm{mSv}$ for a standard 10/30 mCi rest-stress Tc-99m MPI scan. Some laboratories, however, have been successfully performing stress first imaging (SFI) protocol where a "normal" stress obviates the need for the rest portion [converting the nuclear scan essentially to a stress-only imaging (SOI)]. SFI/SOI protocols have recently been shown to be very safe and feasible as compared with a normal stress-rest $\operatorname{scan}^{4}$ or a normal rest-stress scan. ${ }^{5}$ These studies have enrolled over 16,000 patients and have demonstrated excellent comparable results both for interpretability and a very favorable prognosis extending to over 4.5 years follow up. The concept of SFI/SOI imaging is extremely attractive especially when taken in the context that over $70 \%$ of MPI studies performed in nuclear labs are interpreted as "normal"; 6 thus, a substantial portion of rest studies performed could be avoided and by doing so the advantages are obvious.

For a nuclear physician to conclusively label a stress study normal and avoid the rest scan, the study as a whole must be "completely normal" including the stress perfusion images, gated imaging parameters such as ejection fraction $(>50 \%)$, cavity size, and wall motion. Furthermore, stress ECG and planar images must be reviewed, and attenuation correction (AC) or prone imaging should be used if available as an adjunct for the stress study. AC enhances the confidence of the reader to call the stress portion normal as approximately 50-78\% of non-attenuation-corrected images have artifacts. ${ }^{7,8}$ This constellation of findings in a SFI study eliminates the need for a rest study and makes it a SOI study. 
The potential benefits of SFI or SOI study are numerous: faster throughput leading to greater lab efficiency, lower radiation, greater patient satisfaction, and cost savings both from the scan and radiopharmaceutical. The study by Chang et al showed a significant reduction of $60 \%$ in the radiopharmaceutical dose received by patients who had SOI $(21.3 \pm 10.7 \mathrm{mCi})$ vs those who underwent additional rest imaging $(55.1 \pm 11.9 \mathrm{mCi})$. Ideal patients for attempting the SFI or SOI would be those that are low to intermediate likelihood for CAD, able to exercise, have regular rhythm to enable gating for $\mathrm{EF}$ and wall motion assessment and availability of $\mathrm{AC}$ in the lab. However, SFI/SOI has been used in large studies even in patients with prior CAD successfully. ${ }^{4,5}$ Furthermore SOI has been successfully evaluated in obese patients ${ }^{9}$ and in emergency department patients. ${ }^{10}$

A normal SFI/SOI study also has an outstanding prognosis with events rates of $<1 \% /$ year in large retrospective studies of thousands of patients. ${ }^{4,5}$ Most recently, Duvall et $\mathrm{al}^{11}$ have in a different study demonstrated the excellent prognosis following a normal SOI using Thallium protocol compared with a normal SOI using Tc-99m or normal standard rest-stress protocol. Finally, impressive reductions in radiation by modifying SOI and SRI acquisition strategies have been recently published and serve as the "icing" on the cake of SFI/SOI protocols. ${ }^{12,13}$

Why then has SFI/SOI not been more widely adopted by nuclear laboratories? Some challenges when performing SFI/SOI protocols merit discussion. The laboratory logistics and schedules may be thrown out of sync in a one-camera scenario particularly if SFI is done in some patients and rest-stress in others and then a high dose rest study is needed in the SFI patient. If a 8-10 $\mathrm{mCi}$ stress first is done and a subsequent high-dose (24-30 $\mathrm{mCi}$ ) rest imaging is needed, then problems with "shine through" of stress dose into rest images and underestimation of defect reversibility may be of concern to some. In heavier patients, the count statistics in the low-dose stress images may not be great, imposing difficulty in the interpretation of both perfusion and gated images. If SOI images are being done, then it should ideally be accompanied by AC which may not be available in all labs. Information on TID is lacking if $\mathrm{SOI}$ is used in decision making. Immediate processing and interpretation by a physician needs to be done for $\mathrm{SFI} / \mathrm{SOI}$ to decide if rest imaging is needed. A regular rhythm to enable gating to assess EF and wall motion is important too in case of SOI.

Perhaps one of the most important questions is: which patients would be best suited for an SFI strategy where we could reasonably predict that a rest scan can be avoided? In this issue of the Journal, Duvall et al $^{14}$ have devised a simple clinical score in an attempt to identify the right patient population or SFI. The authors have performed a retrospective single-center study where they reviewed Tc-99m SPECT MPI data for a period of 27 months $(n=7,341)$ done at their institution. They used data from the CZT camera as the derivation set to generate a scoring system to identify successful SFI patient characteristics and then applied the prediction score to another group of patients undergoing MPI with standard SPECT camera and CZT camera to validate the score. Pre-test risk was determined using standard ACC/AHA pre-test probability scores. The authors used a summed stress score (SSS) of $\leq 1$ to retrospectively categorize a successful SFI in the derivation cohort with CZT camera (with or without AC using prone imaging, $\mathrm{n}=1,966$ ). Similar criteria were used for their validation cohort on the CZT camera $(\mathrm{n}=1,005)$. For the validation cohort in their regular SPECT camera $(n=2,430)$, they used a SSS of $\leq 3$ for the non-AC patients and $\leq 1$ for the AC patients. The details of their algorithm are indicated in the flow diagram of Fig. 1 of their article. Both exercise and pharmacologic stress modalities were employed. Key characteristics of patients were upto $70 \%$ being outpatients with $65-70 \%$ having normal perfusion on imaging with age range of 60-65 with almost equal distribution of men and women.

The authors identified variables associated with unsuccessful SFI from the derivation cohort and entered these variables with integer-weighting system to be tested in the validation cohort. The variables were age $>65$ years ( 1 point), diabetes ( 2 points), typical chest pain ( 2 points), congestive heart failure (3 points), abnormal ECG (4 points), male gender (5 points), and documented CAD (5 points). Emergency room location interestingly was associated negatively with unsuccessful SFI ( -2 points) which could reflect lower risk chest pain patients in the observation unit getting tested and more likely to have normal SFI. The mean score of study group was $7.2 \pm 5.4$ within a range from -2 to 21 .

The key findings of this study were as follows:

1. A higher prediction score derived from the above variables was associated with unsuccessful SFI. When divided in to low $(-2$ to $<5)$, intermediate $(>5-<10)$, or high $(>10-21)$ scores, there was a steady increase in unsuccessful SFI of $8.1 \%, 26.9 \%$, and $64.7 \%$ in the three categories, respectively.

2. Receiver operating curve analysis showed that the prediction score had a $c$-statistic of 0.82 to predict unsuccessful SFI. A score of 11 points offered a sensitivity (57\%) and specificity (88\%) and predicted the highest $\%$ of patients who were correctly classified $(77.2 \%)$ with regard to the likelihood of unsuccessful SFI. 
3. Low-risk groups were associated with a higher rate of unsuccessful SFI protocol with pharmacologic vs exercise tests $(5.9 \%$ vs $12.1 \%, P=.009)$.

4. Lack of $\mathrm{AC}$ was associated with higher rates of unsuccessful SFI in low- and intermediate-risk tertiles of validation cohort compared with validation cohort patients who had either prone or line source form of AC.

5. Overall, a low prediction score of $(-2$ to $<5)$ predicts high rate of successful SFI in approximately $92 \%$ of patients.

The authors deserve accolades for taking on this effort to further advance the widespread application of SFI/SOI strategies. The authors have extensive experience in this area based on their previous study with SFI/SOI and have proposed a simple prediction score for choosing patients who can undergo SFI.

What are the implications of this article and can we employ this score into our daily practice?

a. Will this score change our management and enhance decision making? In this case, the answer is "yes" as a low score $(-2$ to $<5)$ identifies patients where stress portion has a high likelihood of being normal. Thus, avoiding rest imaging induces cost savings, leads to radiation reduction, and impacts downstream management and disposition. However, the score obviously needs more external validation outside the authors' institution.

b. Can a busy clinician apply this score readily in a way that it is not cumbersome to use? In its current form, the score is easy to use. The clinical variables incorporated are simple, straightforward to remember, and can be easily incorporated into a screening/ triage questionnaire. Moreover, most of these variables in the score are routinely used by many of us in clinical risk prediction on a daily basis. However, the success of using this score is also driven by a team effort where it is very essential to confirm that the entire clinical study is "normal." A dedicated nuclear physician must review the studies soon after the stress portion is done and decide if a rest study is needed. This requires commitment on behalf of the staff and the physicians.

c. Does this score demonstrate good ability to be applied across a unselected group of patients in different settings? "Not yet." The authors have tried to address this issue by retrospectively applying this method to their patient population and validating it in different camera settings. However, its external validity remains to be tested in a prospective fashion in other patient populations.

d. Is there cost/risk to patients involved in implementing it? "No." The worst-case scenario is that the prediction score fails to identify the low-risk patient and a subsequent rest image is done the same day or the next day which is any way part of standard of care. The common sense rule of "when in doubt, do the rest study" is always an option.

As correctly pointed out by the authors, the applicability of this prediction score has some limitations. Prospective external validation is lacking at this juncture. Also as pointed out, male gender gets a pretty high score-just by itself immediately pushing a male into the intermediaterisk category-which likely could have been driven by different attenuation characteristics between males and females when derived using CZT cameras. This could make routine use of this score in males to identify low-risk group a little challenging as addition of one parameter such as abnormal ECG pushes the score to 9 which then predicts higher rates of failure for a successful SFI. The authors addressed this in a way by dropping the weighting factor for male gender from 5 to 4 and showed no significant differences in predictive value of the score.

In conclusion, this initiative by Duvall et al has moved SFI/SOI imaging protocols a step closer to more widespread use and have provided us with a systematic scoring tool and reassurance to those of us who tend to apply different components of this score on an individual basis to choose the right patient for SFI. Furthermore, we believe that AC either with line source, $\mathrm{CT}$, or prone imaging is a key component in the success of SFI/SOI protocols particularly for rapid and confident decision making. Time has come to put to "rest" the routine rest imaging in all patients either before or after stress imaging by adopting simple strategies such as this prediction score. Let us capitalize on normal stress images, its diagnostic and prognostic value, and at the same time reduce cost and radiation and enhance patient satisfaction. It is a "win-win" situation if executed well.

\section{References}

1. Lucas FL, DeLorenzo MA, Siewers AE, Wennberg DE. Temporal trends in the utilization of diagnostic testing and treatments for cardiovascular disease in the United States, 1993-2001. Circulation 2006;113:374-9.

2. Fazel R, Krumholz HM, Wang Y, Ross JS, et al. Exposure to low dose ionizing radiation from medical procedures. N Engl J Med 2009;361:849-57.

3. Depuey EG, Mahmarian JJ, Miller TD, Einstein AJ, et al. Patient centered imaging. J Nucl Cardiol 2012;19:185-215.

4. Chang SM, Nabi F, Xu J, Raza U, et al. Normal stress-only versus standard stress/rest myocardial perfusion imaging: similar patient mortality with reduced radiation exposure. J Am Coll Cardiol 2010;55:221-30.

5. Duvall WL, Wijetunga MN, Klein TM, Razzouk L, et al. The prognosis of a normal stress-only Tc-99m myocardial perfusion imaging study. J Nucl Cardiol 2010;17:370-7. 
6. Hachamovitch R, Berman DS, Shaw LJ, Kiat H, et al. Incremental prognostic value of myocardial perfusion single photon emission computed tomography for the prediction of cardiac death: differential stratification for risk of cardiac death and myocardial infarction. Circulation 1998;98(2):190.

7. Singh B, Bateman TM, Case JA, Heller G. Attenuation artifact, attenuation correction, and the future of myocardial perfusion SPECT. J Nucl Cardiol 2007;14:153-64.

8. Heller GV, Bateman TM, Johnson LL, Cullom SJ, et al. Clinical value of attenuation correction in stress-only Tc-99m sestamibi SPECT imaging. J Nucl Cardiol 2004;11:273-81.

9. Gemignani AS, Muhlebach SG, Abbott BG, Roye GD, et al. Stress-only or stress/rest myocardial perfusion imaging in patients undergoing evaluation for bariatric surgery. J Nucl Cardiol 2011; 18:886-92.

10. Gibson PB, Demus D, Noto R, Hudson W, et al. Low event rate for stress-only perfusion imaging in patients evaluated for chest pain. J Am Coll Cardiol 2002;39:999-1004.
11. Duvall WL, Hiensch RJ, Levine EJ, Croft LB, et al. The prognosis of a normal T1-201 stress-only SPECT MPI study. J Nucl Cardiol 2012; doi:10.1007/s12350-012-9601-2.

12. Bateman TM, Heller GV, McGhie AI, Courter SA, et al. Multicenter investigation comparing a highly efficient half-time stressonly attenuation correction approach against standard rest-stress Tc-99m SPECT imaging. J Nucl Cardiol 2009;16:726-35.

13. Depuey EG, Ata P, Wray R, Friedman M. Very low-activity stress/ high-activity rest, single-day myocardial perfusion SPECT with a conventional sodium iodide camera and wide beam reconstruction processing. J Nucl Cardiol 2012; doi:10.1007/s12350-012-9596-8.

14. Duvall WL, Baber U, Elliot L, Croft L, et al. A model for successful prediction of stress-first Tc-99m MPI. J Nucl Cardiol 2012; doi:10.1007/s12350-012-9606-x. 